\title{
Uniformly Best Constant Risk and Minimax Point Estimates ${ }^{1}$
}

\author{
Raymond P. Peterson
}

\begin{abstract}
In this paper several types of point estimates are compared on the bases of their corresponding expected risk. It is shown that constant risk minimax estimates (which are always uniformly best constant risk estimates) exist, under certain conditions, for several frequently occurring types of parameters and general methods are obtained for constructing these minimax estimates.
\end{abstract}

\section{Introduction}

Let $x_{1}, \ldots, x_{n}$ denote $n$ (not necessarily independent) observed values of a random variable $\xi$, which is distributed over a space $S$ according to a distribution $P_{\xi}\left(x, \theta_{1}, \ldots, \theta_{s}\right)$. It is assumed that $P_{\xi}(x$, $\left.\theta_{1}, \ldots, \theta_{s}\right)$ is completely specified except for the $s$ unknown parameters $\theta_{1}, \ldots, \theta_{s}$. These parameters may be represented by a point $\theta=\left(\theta_{1}, \ldots\right.$., $\left.\theta_{s}\right)$ in the $s$-dimensional Euclidean parameter space $\Omega$. Also $X=\left(x_{1}, \ldots, x_{n}\right)$ is a point in the $n$-dimensional Euclidean sample space, $M$. We shall assume that $P_{\xi}(x, \theta)$ is absolutely continuous, that is, $\xi$ possesses an integrable probability density function $g_{\xi}(x, \theta)$. Let $p(X, \theta)=p\left(x_{1}, \ldots, x_{n}, \theta\right)$ denote the joint probability density function of the observations at $X \epsilon M$.

A statistical point estimate of a parameter $\theta_{i}$. which ranges over a subset $\omega_{i}$ of one-dimensional Euclidean space, is a function $f_{i}(X)$ of the sample values that takes on values in $\omega_{i}$. Let $W\left[f_{i}(X), \theta\right]$ be a nonnegative measurable function defined for all $\theta \epsilon \Omega$ and $X \epsilon M$. $W\left[f_{i}(X), \theta\right]$ is a weight function that represents the relative seriousness of taking $f_{i}(X)$ as the value of $\theta_{i}$ for any particular sample point $X$. The function

$$
r_{f_{i}}(\theta)=\int_{M} W\left[f_{i}(X), \theta\right] p(X, \theta) d X
$$

represents the risk or expected loss incurred by using $f_{i}(X)$ to estimate $\theta_{i}$ when $\theta$ is the true parameter point. Thus $r_{f_{i}}(\theta)$ is defined as the risk function of $f_{i}(X)$. The expected risk of $f_{i}(X)$, relative to an $a$ priori distribution $\lambda(\theta)$ of $\theta$ is given by

$$
R_{f_{i}}(\boldsymbol{\lambda})=\int_{\Omega} \int_{M} W\left[f_{i}(X), \theta\right] p(X, \theta) d X d \lambda(\theta) .
$$

We can now define the following classes of point estimates in terms of $r_{f_{i}}(\theta)$ and $R_{f_{i}}(\lambda)$.

(1) A minimax estimate of $\theta_{i}$ is one which minimizes $\sup r_{f_{i}}(\theta)$.

\footnotetext{
1. Most of the work in this paper was done while the author was a Research Fellow at the Institute for Numerical Analysis, National Bureau of Standards, Fellow at the Institute of Naval Research. Author's present address is University of Washington, Seattle.
}

(2) A constant risk (CR) estimate $f_{i}$ of $\theta_{i}$ is one such that $r_{f_{i}}(\theta)$ is constant.

(3) A Bayes estimate of $\theta_{i}$, relative to an a priori distribution $\bar{\lambda}(\theta)$, is one that minimizes $R_{f_{i}}(\bar{\lambda})$.

(4) A uniformly best (UB) estimate of $\theta_{i}$ is one that minimizes $R_{f_{i}}(\lambda)$ for all possible a priori distributions $\lambda(\theta)$.

(5) A uniformly best constant risk (UBCR) estimate of $\theta_{i}$ is one that is a UB-estimate among all CRestimates.

(6) A constant risk minimax (CRM) estimate is an estimate that is both a CR and a minimax estimate.

It is evident that a UB-estimate is preferable to any other, provided that one can be obtained. We will show that in several important cases it is reasonable to restrict our choice to CRM-estimates, since they possess certain desirable properties and, in most cases, are relatively easy to obtain. The concepts of a risk function, expected risk, and minimax estimates used here are due to Wald [ [5 to 8].

Let

$$
\varphi_{f_{i}}(X)=\int_{\Omega} W\left[f_{i}(X), \theta\right] p(X, \theta) d \theta .
$$

Theorem 1.1 Let $f_{i}(X)$ be a $C R$ estimate of $\theta_{i}$ and suppose that for any other estimate $\bar{f}_{i}(X)$ there exists a probability measure $\bar{\lambda}(\theta)$ over $\Omega$ such that

$$
\int_{\Omega} r_{f_{i}}(\theta) d \bar{\lambda}(\theta) \leq \int_{\Omega} r_{\bar{f}_{i}}(\theta) d \bar{\lambda}(\theta) .
$$

\section{Then $f_{i}(X)$ is a minimax estimate.}

Proof. Let $\bar{f}_{i}(X)$ be any other estimate and let $\bar{\lambda}(\theta)$ be a probability measure such that (2) is satisfied. Then

$$
\begin{aligned}
c-\sup _{\theta} r_{\bar{f}_{i}}(\theta) & \leq \int_{\Omega}\left[c-r_{\bar{f}_{i}}(\theta)\right] d \bar{\lambda}(\theta) \\
& \leq \int_{\Omega}\left[r_{f_{i}}(\theta)-r_{\bar{f}_{i}}(\theta)\right] d \bar{\lambda}(\theta) \leq 0
\end{aligned}
$$

where $r_{f_{i}}(\theta) \equiv c$ ( $c$ a constant). Therefore

$$
c=\sup _{\theta} r_{f_{i}}(\theta) \leq \sup _{\theta} r_{\bar{f}_{i}}(\theta),
$$

and the theorem follows. 
Corollary 1.1 Any CR-estimate which is a Bayes estimate relative to some probability measure $\lambda^{\prime}(\theta)$ is a minimax estimate.

Proof. This corollary follows immediately from theorem 1.1.

Theorem 1.2. Any CR-estimate $f_{i}(X)$ that is a minimax estimate is a UBCR-estimate.

Proof. Let $\bar{f}_{i}(X)$ be any other CR-estimate. Then

$$
\begin{aligned}
R_{\bar{f}_{i}}(\lambda)-R_{f_{i}}(\lambda) & =\int_{\Omega}\left[r_{\bar{f}_{i}}(\theta)-r_{f_{i}}(\theta)\right] d \lambda(\theta) \\
& =\bar{c}-c=\sup _{\theta} r_{\bar{f}_{i}}(\theta)-\sup _{\theta} r_{f_{i}}(\theta) \geq 0,
\end{aligned}
$$

where $\lambda(\theta)$ is any probability measure over $\Omega$, $r_{f_{i}}(\theta) \equiv \bar{c}, \quad r_{f_{i}}(\theta) \equiv c, \quad(c$ and $\bar{c}$ constants $)$. Thus $f_{i}(X)$ is a UBCR-estimate.

Theorem 1.3. Suppose that a CR-estimate $f_{i}(X)$ minimizes $\phi_{f_{i}}(X)$ for all $X \epsilon M$ and that at least one of the following conditions $(A)$ and $(B)$ is satisfied:

(A) $\Omega$ is compact

(B) $r_{f_{i}}(\theta)$ and $\phi_{f_{i}}(X)$ are uniformly convergent over $M$ and $\Omega$, respectively. Then $f_{i}(X)$ is both a minimax and a UBCR-estimate of $\theta_{i}$.

Proof. Let $\bar{f}_{i}(X)$ be any other estimate of $\theta_{i}$. Then

$$
\phi_{f_{i}}^{-}(X)-\phi_{f_{i}}(X) \geq 0
$$

for all $X \epsilon M$. Let $M_{q}$ and $\Omega_{j}$ be compact subsets of positive measure of $M$ and $\Omega$, respectively, such that

$$
\begin{array}{ll}
M_{q}<M_{q+1}, & q=1,2, \ldots \\
\Omega_{j}<\Omega_{j+1}, & j=1,2, \ldots
\end{array}
$$

and

$$
\begin{gathered}
\lim _{q=\infty} M_{q}=M, \\
\lim _{j=\infty} \Omega_{j}=\Omega .
\end{gathered}
$$

Since $W\left[f_{i}(X), \theta\right]$ is nonnegative and measurable, it follows from Fubini's Theorem (see, for example, [1] or [4]) that

$$
\begin{aligned}
& \int_{M_{q}} \int_{\Omega_{j}} W\left[f_{i}(X), \theta\right] p(X, \theta) d \theta d X \\
= & \int_{\Omega_{i}} \int_{M_{q}} W\left[f_{i}(X), \theta\right] p(X, \theta) d X d \theta
\end{aligned}
$$

for all $j$ and $q$. By hypothesis,

$$
r_{f_{i}}(\theta) \equiv c,
$$

where $c$ is a constant.

Suppose first that condition (A) is satisfied; that is, $\Omega$ is compact. Then from (4),

$$
\begin{aligned}
& \int_{M} \int_{\Omega} W\left[f_{i}(X), \theta\right] p(X, \theta) d \theta d X \\
= & \int_{\Omega} \int_{M} W\left[f_{i}(X), \theta\right] p(X, \theta) d X d \theta .
\end{aligned}
$$

Since $\Omega$ is compact,

$$
\left.\begin{array}{rl} 
& \int_{M}\left[\phi_{\bar{f}_{i}}(X)-\phi_{f_{i}}(X)\right] d X=\int_{\Omega}\left[r_{\bar{f}_{i}}(\theta)-r_{f_{i}}(\theta)\right] d \theta \\
= & \int_{\Omega}\left[r_{\bar{f}_{i}}(\theta)-c\right] d \theta \leq\left[\sup _{\theta} r_{\bar{f}_{i}}(\theta)-c\right] \int_{\Omega} d \theta .
\end{array}\right\}
$$

But, it follows from (3) that the first integral in (5) is nonnegative and therefore

$$
\sup r_{\bar{f}_{i}}(\theta) \geq c \text {. }
$$

Now suppose that $\Omega$ is not compact, but condition (B) is satisfied. From (3) it is seen that either

$$
\phi_{\bar{f}_{i}}^{-}(X)-\phi_{f_{i}}(X) \equiv 0
$$

for all $X \epsilon M$ or

$$
\phi_{\bar{f}_{i}}(X)-\phi_{f_{i}}(X)>\epsilon>0
$$

over some set $M^{\prime}$ in $M$, where $M^{\prime}$ has finite positive measure $\mathrm{m}^{\prime}$.

First, consider the.case where (7a) holds. Let $M_{q}$ always be taken so that $M^{\prime}<M_{q}$. Then, from (3), (4), and (7a), it follows that

$$
\begin{aligned}
& \lim _{j=\infty} \int_{M_{q}} \int_{\Omega_{j}}\left\{W\left[\bar{f}_{i}(X), \theta\right]-W\left[f_{i}(X), \theta\right]\right\} p(X, \theta) d \theta d X \\
& =\int_{M_{q}} \int_{\Omega}\left\{W\left[\bar{f}_{i}(X), \theta\right]-W\left[f_{i}(X), \theta\right]\right\} p(X, \theta) d \theta d X \\
& =\int_{M_{q}}\left[\phi_{\bar{f}_{i}}(X)-\phi_{f_{i}}(X)\right] d X>\epsilon m^{\prime} .
\end{aligned}
$$

Let $\bar{W}_{i}-W_{i}=W\left[\bar{f}_{i}(X), \theta\right]-W\left[f_{i}(X), \theta\right]$. Since, by hypothesis,

$$
\int_{M_{q}}\left[\bar{W}_{i}-W_{i}\right] p(X, \theta) d X
$$

is uniformly convergent over $M$, there exists a $q_{0}$ such that

$$
\left.\begin{array}{rl}
\mid \int_{M_{q}}\left[\bar{W}_{i}-W_{i}\right] p(X, \theta) d X \\
\quad-\int_{M}\left[\bar{W}_{i}-W_{i}\right] p(X, \theta) d X \mid<\frac{\epsilon}{2}, q \geq q_{0}
\end{array}\right\}
$$

Also since, by hypothesis,

$$
\int_{\Omega_{j}}\left[\bar{W}_{i}-W_{i}\right] p(X, \theta) d \theta
$$


is uniformly convergent over $\Omega$, there exists a $j_{0}$ such that

$$
\left.\begin{array}{l}
\mid \int_{\Omega_{j}}\left[\bar{W}_{i}-W_{i}\right] p(X, \theta) d \theta \\
-\int_{\Omega}\left[\bar{W}_{i}-W_{i}\right] p(X, \theta) d \theta \mid<\frac{\epsilon m^{\prime}}{2 m_{q_{0}}}, j \geq j_{0},
\end{array}\right\}
$$

where $m_{q_{0}}$ is the measure of the set $M_{q_{0}}$. Then, from (7a) and (9) it follows that

$$
\begin{aligned}
& \int_{\Omega_{\Omega_{0}}} \int_{M_{q_{0}}}\left[\bar{W}_{i}-W_{i}\right] p(X, \theta) d X d \theta \\
= & \int_{M_{q_{0}}} \int_{\Omega_{j_{0}}}\left[\bar{W}_{i}-W_{i}\right] p(X, \theta) d \theta d X \\
= & \int_{M_{q_{0}}}\left[\int_{\Omega}\left[\bar{W}_{i}-W_{i}\right] p(X, \theta) d \theta+\alpha \frac{\epsilon m^{\prime}}{2 m_{q_{0}}}\right] d X, \quad|\alpha| \leq 1, \\
= & \int_{M_{q_{0}}} \int_{\Omega}\left[\bar{W}_{i}-W_{i}\right] p(X, \theta) d \theta d X+\alpha \frac{\epsilon m^{\prime}}{2} \\
& >\epsilon m^{\prime}-\frac{\epsilon m^{\prime}}{2}=\frac{\epsilon m^{\prime}}{2} .
\end{aligned}
$$

Therefore

$$
\int_{\Omega j_{0}} \int_{M_{q_{0}}}\left[\bar{W}_{i}-W_{i}\right] p(X, \theta) d X d \theta>\frac{\epsilon m^{\prime}}{2} .
$$

From (8) and (10) we have that

$\int_{\Omega_{0}}\left[\int_{M}\left[\bar{W}_{i}-W_{i}\right] p(X, \theta) d X+\beta \cdot \frac{\epsilon}{2}\right] d \theta>\frac{\epsilon m^{\prime}}{2},|\beta|<\mid 1$,

and thus

$$
\left.\begin{array}{r}
{\left[\left(\sup _{\theta} r_{f_{i}}(\theta)-c\right)+\beta \cdot \frac{\epsilon}{2}\right] \int_{\Omega_{j_{n}}} d \theta} \\
\geqq \int_{\Omega_{j_{0}}}\left[\left(r_{f_{i}}(\theta)-c\right)+\beta \cdot \frac{\epsilon}{2}\right] d \theta>\frac{\epsilon m^{\prime}}{2}>0 .
\end{array}\right\}
$$

If $\sup r_{\bar{f}_{i}}(\theta)-c<0$, (11) is impossible since $\epsilon$ is arbitrarily small, and therefore

$$
\sup _{\theta} r_{f_{i}}(\theta)-c \geq 0 .
$$

The proof that (12) is true for the case in which (7) holds is immediate. Hence, since $c \equiv \sup r_{\bar{f}_{i}}(\theta)$, $f_{i}(X)$ is a minimax estimate and by Theorem ${ }^{\theta}$. 2 is also a UBCR-estimate.

\section{Classes of CR-Estimates}

In this section we shall find classes of CR-estimates for several frequently occurring types of parameters.

\subsection{L-Estimates of a Location Parameter}

If $p(X, \theta)$ can be expressed in the form

$$
p\left(x_{1}-\theta, \ldots, x_{n}-\theta\right)
$$

then $\theta$ is called a location parameter. An estimate $f\left(x_{1}, \ldots ., x_{n}\right)$ will be called an L-estimate of the location parameter $\theta$ provided

$$
f\left(x_{1}+\tau, \ldots, x_{n}+\tau\right)=f\left(x_{1}, \ldots, x_{n}\right)+\tau
$$

for any real $\tau$.

\subsection{S-Estimates of a Scale Parameter}

If $p(X, \theta)$ can be expressed in the form

$$
\theta^{-n} p\left(\frac{x_{1}}{\theta}, \ldots, \frac{x_{n}}{\theta}\right), \quad \theta \geq 0,
$$

then $\theta$ is called a scale parameter. An estimate $f\left(x_{1}, \ldots ., x_{n}\right)$ will be called an S-estimate of the scale parameter $\theta$ provided

$$
f\left(\mu x_{1}, \ldots, \mu x_{n}\right)=\mu f\left(x_{1}, \ldots, x_{n}\right), \quad \mu \geq 0
$$
and

$$
f\left(x_{1}, \ldots, x_{n}\right) \geq 0 .
$$

\section{3. $L(S)$-Estimates of a Location Parameter (Scale Parameter Unknown)}

Suppose $p(X, \theta)$ is of the form

$$
\theta_{2}^{-n} p\left(\frac{x_{1}-\theta_{1}}{\theta_{2}}, \ldots, \frac{x_{n}-\theta_{1}}{\theta_{2}}\right), \quad \theta_{2} \geq 0,
$$

where $\theta_{1}$ and $\theta_{2}$ are unknown parameters. Then an estimate $f_{1}\left(x_{1}, \ldots, x_{n}\right)$ will be called an $L(S)$ estimate of the location parameter $\theta_{1}$ (the scale parameter $\theta_{2}$ unknown) provided that $f_{1}(X)$ is an $L$-tstimate, that is, (14) is satisfied, and also

$$
f_{1}\left(\mu x_{1}, \ldots, \mu x_{n}\right)=\mu f_{1}\left(x_{1}, \ldots, x_{n}\right)
$$

for any real $\mu$.

\section{4. $S(L)$-Estimates of a Scale Parameter (Location Parameter Unknown)}

Let $p(X, \theta)$ be of the form

$$
\theta_{2}^{-n} p\left(\frac{x_{1}-\theta_{1}}{\theta_{2}}, \ldots, \frac{x_{n}-\theta_{1}}{\theta_{2}}\right), \quad \theta_{2} \geq 0
$$

where $\theta_{1}$ and $\theta_{2}$ are unknown parameters. Then an estimate $f_{2}\left(x_{1}, \ldots ., x_{n}\right)$ is called an $S(L)$-estimate of the scale parameter $\theta_{2}$ (the location parameter $\theta_{1}$ unknown) provided that $f_{2}(x)$ is an $S$-estimate, that is, $f_{2}(X)$ satisfies (16) and (17), and also

$$
f_{2}\left(x_{1}+\tau, \ldots, x_{n}+\tau\right)=f_{2}\left(x_{1}, \ldots, x_{n}\right)
$$

for all real $\tau$.

\subsection{D-Estimates of the Difference Between Two Location Parameters}

Let $p(X, Y, \theta, \delta)$ be the joint probability density function of $x_{1}, \ldots ., x_{n}$ and $y_{1}, \ldots, y_{n}$, where 
$X$ and $Y$ are samples from two populations with unknown location parameters $\theta$ and $\theta+\delta$, respectively. Then $p(X, Y, \theta, \delta)$ is of the form

$$
p\left(x_{1}-\theta, \ldots, x_{m}-\theta, y_{1}-\theta-\delta, \ldots, y_{n}-\theta-\delta\right) \text {. }
$$

An estimate $f(X, Y)$ will be called a D-tstimate of the difference $\delta$ provided

$$
\begin{aligned}
& f\left(x_{1}+\mu, \ldots, x_{m}+\mu, y_{1}+\lambda, \ldots, y_{n}+\lambda\right) \\
= & f\left(x_{1}, \ldots, x_{m}, y_{1}, \ldots, y_{n}\right)+(\lambda-\mu)
\end{aligned}
$$

for all real $\mu$ and $\lambda$.

\section{6. $R$-Estimates of the Ratio of Two Scale Parameters}

Let $p(X, Y, \theta, \rho)$ be of the form

$\theta^{-m}(\rho \theta)^{-n} p\left(\frac{x_{1}}{\theta}, \ldots, \frac{x_{m}}{\theta}, \frac{y_{1}}{\rho \theta}, \ldots, \frac{y_{n}}{\rho \theta}\right), \quad \theta, \rho>0$

where $X$ and $Y$ are samples from two populations with unknown scale parameters $\theta$ and $\rho \theta$, respectively. An estimate $f(X, Y)$ is called an $R$-estimate of the ratio $\rho$ provided

$$
\begin{aligned}
& f\left(\mu x_{1}, \ldots, \mu x_{m}, \lambda y_{1}, \ldots, \lambda y_{n}\right) \\
= & \frac{\lambda}{\mu} f\left(x_{1}, \ldots, x_{m}, y_{1}, \ldots, y_{n}\right)
\end{aligned}
$$

for all $\mu, \lambda>0$

We now show that any estimate belonging to one of the classes 2.1 to 2.6 is a CR-estimate provided that the weight function $W_{i}$ is of proper form. The following six theorems are stated as one.

Theorems 2.1 to 2.6. Let the density function $p$ be of the form given in classes 2.1 to 2.6 and let the weight function $W_{i}$ be of the form (1) $W[f(X)-\theta]$, (2) $W\left[\theta^{-1} f(X)\right], \quad(3) W\left[\theta_{2}^{-1}\left(f_{1}(X)-\theta_{1}\right)\right]$, (4) $W\left[\theta_{2}^{-1}\right.$ $\left.f_{2}(X)\right]$, (5) $W[f(X, Y)-\delta],(6) W\left[p^{-1} f(X, Y)\right]$. Then, if $f_{i}$ is an (1) $L$, (2) $S$, (3) $L(S)$, (4) $S(L)$, (5) D, (6) R-estimate, the risk function $r_{f_{i}}(\theta)$ is constant.

Proof. We shall prove only theorem 2.3, as the others are proved in exactly the same manner. Consider the risk function

$$
\begin{aligned}
r_{f_{i}}\left(\theta_{1}, \theta_{2}\right)= & \int_{-\infty}^{\infty} \ldots \int_{-\infty}^{\infty} W\left[\frac{\left(f_{1} X\right)-\theta_{1}}{\theta_{2}}\right] \theta_{2}^{-n} \\
& \cdot p\left(\frac{x_{1}-\theta_{1}}{\theta_{2}}, \ldots, \frac{x_{n}-\theta_{1}}{\theta_{2}}\right) d x_{1} \ldots d x_{n} .
\end{aligned}
$$

Let

$$
t_{i}=\frac{x_{i}-\theta_{1}}{\theta_{2}},(i=1,2, \ldots, n) .
$$

Since $f_{1}(X)$ is an $L(S)$-estimate

$$
\begin{aligned}
\frac{f_{1}(X)-\theta_{1}}{\theta_{2}} & =\frac{f_{1}\left(x_{1}, \ldots, x_{n}\right)-\theta_{1}}{\theta_{2}}=f_{1}\left(\frac{x_{1}-\theta_{1}}{\theta_{2}}, \ldots, \frac{x_{n}-\theta_{1}}{\theta_{2}}\right) \\
& =f_{1}\left(t_{1}, \ldots t_{n}\right) .
\end{aligned}
$$

Thus, making the transformation (27) and using (28) in (26), we have $r_{f_{\imath}}(\theta)=\int_{-\infty}^{\infty} \cdots \int_{-\infty}^{\infty} W\left[f_{1}\left(t_{1}, \ldots, t_{n}\right)\right] p\left(t_{1}, \ldots, t_{n}\right) d t_{1} \ldots d t_{n}$ which is completely independent of $\theta$ and therefore $r_{f_{i}}(\theta)$ is constant.

\section{CR-Minimax Estimates}

As a direct consequence of Theorem 1.3 and Theorems 2.1 to 2.6 we have the following six theorems which are stated as one.

Theorems 3.1 to 3.6. If at least one of conditions $(A)$ and $(B)$ in Theorem 1.3 is satisfied and if the weight function $W_{i}$ is of the form (1) $W[f(X)-\theta]$, (2) $W\left[\theta^{-1} f(X)\right],(3) W\left[\theta_{2}^{-1}\left(f_{1}(X)-\theta_{1}\right)\right],(4) W\left[\theta_{2}^{-1} f_{2}(X)\right]$, (5) $W[f(X, Y)-\delta],(6) W\left[\rho^{-1} f(X, Y)\right]$, then any (1) $L$, (2) $S$, (3) $L(S)$, (4) $S(L)$, (5) D, (6) R-estimate which minimizes $\left(\phi_{f_{i}} X\right)[$ as defined by formula (1)] for all $X$, $(X, Y) \epsilon M$ is a minimax (and also a $U B C R$ ) estimate of $\theta_{i}$.

Conversely, it has been shown by Kallianpur [3] that, "under mild restrictions", the minimax estimate in the above cases minimizes $\phi_{f_{i}}$ and also belongs to the corresponding class of CR-estimates. For example, in case 3.3 , the minimax estimate of $\theta_{i}$ minimizes $\phi_{f_{i}}(X)$ given in Theorem 3.3 and is an L(S) estimate.

\section{Determination of General Classes of CR-Estimates}

Suppose the joint probability density function $p(X, \theta)$ is of the form

$$
p(X, \theta)=h\left(\eta\left(x_{1}, \theta\right), \ldots, \eta\left(x_{n}, \theta\right)\right) \prod_{i=1}^{n} \frac{\partial \eta\left(x_{i}, \theta\right)}{\partial x_{i}},
$$

where $\eta\left(x_{i}, \theta\right)$ possesses the $n$ first partial derivatives $\partial \eta\left(x_{i}, \theta\right) / \partial x_{i}$ continuous in $x_{i}, \quad(i=1,2, \ldots, n)$. Let $M$ be an $n$-dimensional interval $\left(a_{i} \leq x_{i} \leq b_{i}\right)$ such that

$$
\left.\begin{array}{l}
\eta\left(a_{i}, \theta\right) \equiv c_{i} \\
\eta\left(b_{i}, \theta\right) \equiv d_{i}, \quad(i=1,2, \ldots, n)
\end{array}\right\}
$$

where the $a_{i}, b_{i}, c_{i}$ and $d_{i}$ are constants (possibly infinite) which are independent of $\theta$. Let $\zeta(x, y)$ and $\psi(\theta)$ be arbitrary functions such that the weight function $V\left[\zeta\left(f_{i}(X), \quad \psi(\theta)\right)\right]$ is non-negative and measurable over the product space $M X \Omega$. Then we define the risk function of an estimate $f_{i}(X)$ of $\theta_{i}$ to be

$$
\begin{aligned}
r_{f_{i}}(\theta)= & \int_{M} V\left[\zeta\left(f_{i}(X), \psi(\theta)\right)\right] p(X, \theta) d X \\
= & \int_{M} V\left[\zeta\left(f_{i}(X), \psi(\theta)\right)\right] h\left(\eta\left(x_{1}, \theta\right), \ldots,\right. \\
& \left.\eta\left(x_{n}, \theta\right)\right) \prod_{i=1}^{\mathrm{n}} \frac{\partial \eta\left(x_{i}, \theta\right)}{\partial x_{1}} d x_{i} .
\end{aligned}
$$


The following theorem yields a method for determining general classes of CR-estimates, that is, estimates that possess constant risk functions. that

Theorem 4.1. If $f_{i}(X)=f_{i}\left(x_{1}, \ldots, x_{n}\right)$ is such

$$
f_{i}\left(\eta\left(x_{1}, \theta\right), \ldots, \eta\left(x_{n}, \theta\right)\right)=\zeta\left(f_{i}(X), \psi(\theta)\right)
$$

then the risk function $r_{f_{i}}(\theta)$ is constant.

Proof. Let $f_{i}(X)$ be any estimate satisfying (31) and let

$$
t_{i}=\eta\left(x_{i}, \theta\right), \quad(i=1,2, \ldots, n) .
$$

Then, from (31) and (32) we have

$$
\zeta\left(f_{i}(X), \psi(\theta)\right)=f_{i}\left(t_{1}, \ldots, t_{n}\right) .
$$

Let $T$ denote the $n$-dimensional interval $\left(c_{i} \leq t_{i} \leq d_{i}\right)$. Applying the transformation (32) and using (29) and (33) in (30) we have

$$
\begin{aligned}
r_{f_{i}}(\theta)= & \int_{M} V\left[\zeta\left(f_{i}(X), \psi(\theta)\right)\right. \\
& . h\left(\eta\left(x_{1}, \theta\right), \ldots, \eta\left(x_{n}, \theta\right)\right) \underset{i=1}{I} \frac{\partial \eta\left(x_{i}, \theta\right)}{\partial x_{i}} d x_{i} \\
= & \int_{T} V\left[f_{i}\left(t_{1}, \ldots, t_{n}\right)\right] h\left(t_{1}, \ldots, t_{n}\right) d t_{1} \ldots d t_{n} \equiv C
\end{aligned}
$$

where $C$ is a constant.

\section{Examples}

As an example to illustrate the usefulness of theorem 4.1, let

$$
p(X, \theta)=\frac{1}{(2 \pi)^{n / 2}} e^{-\frac{1}{2}}{ }_{i} \sum_{=1}^{n}\left(x_{i}-\theta\right)^{2}
$$

and choose $V=(f-\theta)^{2}$. In this case we choose

$$
\eta\left(x_{i}, \theta\right) \equiv x_{i}-\theta, \psi(\theta) \equiv \theta, \quad \zeta(f(X), \psi(\theta)) \equiv f(X)-\theta .
$$

Then (31) becomes

$$
f\left(x_{1}-\theta, \ldots, x_{n}-\theta\right)=f\left(x_{1}, \ldots, x_{n}\right)-\theta .
$$

Thus, for example, any weighted mean $\sum_{i=1}^{n} a_{i} x_{i}$, $\sum_{i=1}^{n} a_{i}=1$ is a CR-estimate of $\theta$.

If we take

$$
p(X, \theta)=\frac{1}{(2 \pi \theta)^{n / 2}} e^{-\frac{1}{2}}{ }_{i=1}^{n} \frac{x_{i}^{2}}{\theta}, \theta>0
$$

and choose

$$
V=\left(\frac{f-\theta}{\theta}\right)^{2}=\left(\frac{f}{\theta}-1\right)^{2}
$$

then we can take

$$
\eta\left(x_{i}, \theta\right) \equiv \frac{x_{i}}{\theta^{1 / 2}}, \quad \psi(\theta) \equiv \theta, \quad \zeta(f(x), \psi(\theta)) \equiv \frac{f}{\theta}
$$

and (31) becomes

$$
f\left(\frac{x_{1}}{\theta^{1 / 2}}, \ldots, \frac{x_{n}}{\theta^{1 / 2}}\right)=\frac{f\left(x_{1}, \ldots, x_{n}\right)}{\theta} .
$$

On the other hand, if we are estimating $\theta^{1 / 2}$ by $f(X)$, we take $\psi(\theta) \equiv \theta^{1 / 2}$ and (31) becomes

$$
f\left(\frac{x_{1}}{\theta^{1 / 2}}, \ldots, \frac{x_{n}}{\theta^{1 / 2}}\right)=\frac{f\left(x_{1}, \ldots, x_{n}\right)}{\theta^{1 / 2}} .
$$

In these examples, conditions (34), (35), and (36) show the reasonableness and generality of the respective classes of CR-estimates.

Theorem 4.1 together with Theorem 1.3 can be used to obtain many results similar to Theorems 3.1 to 3.6 , that is, to construct CR-minimax estimates and to throw light on their general desirability. The lower bound for $R_{f_{i}}(\lambda)$, where $f_{i}$ is any CR-estimate, is readily seen to be $r_{f_{i}}(\theta)$, where $\bar{f}_{i}$ is a CR-minimax estimate. This lower bound is

$$
R_{\bar{f}_{i}}(\lambda)=\int_{\Omega} r_{\bar{f}_{i}}(\theta) d \lambda(\theta) \equiv \bar{c},
$$

where $\bar{c}$ is a constant.

In a recent paper, [2], Hodges and Lehmann have illustrated some properties of minimax estimates. They mention, for example, that it has not been possible to obtain a general comparison between minimax estimates and unbiased estimates with uniformly smallest variance, if such exist. We can, imposing certain restrictions on the form of the probability density function $p(X, \theta)$, obtain the CRminimax estimates with uniformly minimum variance and show that these estimates are unbiased. Also, a relationship exists between CR-minimax and maximum likelihood estimates.

\section{References}

[1] P. R. Halmos, Measure theory, p. 147 (D. Van Nostrand Co., Inc., New York, N. Y., 1950).

[2] J. L. Hodges and E. L. Lehmann, Some problems in minimax point estimation, Annals of Math. Stat. 21, 182 to 197 (1950).

[3] G. Kallianpur, Minimax estimates of location and scale parameters, (Abstract), Annals of Math. Stat. 21, 310 (1950).

[4] S. Saks, Theory of the integral, 2d revised ed. p. 77 (Hafnir Pub. Co., New York, N. Y., 1947).

[5] A. Wald, Contributions to the theory of statistical estimation and testing hypotheses, Annals of Math. Stat. 10, 299 to 326 (1939).

[6] A. Wald, Statistical decision functions which minimize the maximum risk, Annals of Math. 46, 265 (1945).

[7] A. Wald, Statistical decision functions, Annals of Math. Stat. 20, 165 (1949).

[8] A. Wald, Statistical decision functions (John Wiley \& Sons, Inc., New York, N. Y., 1950).

Los Avgeles, June 1, 1951. 